\title{
In-bed vital signs monitoring system based on unobtrusive magnetic induction method with a concentric planar gradiometer
}

\author{
Hadiseh Mahdavi and Javier Rosell-Ferrer \\ Group of Biomedical and Electronic Instrumentation, Department of Electronic \\ Engineering, Universitat Politècnica de Catalunya (UPC), Barcelona, Spain \\ E-mail: hadiseh.mahdavi@upc.edu
}

Received 30 November 2016, revised 12 March 2017

Accepted for publication 29 March 2017

Published

\begin{abstract}
Unobtrusive vital signs monitoring is of major importance for various medical areas such as detection and treatment of sleep disorders, monitoring neonates and burned victims, home health care and smart home applications and wearables among others. Such applications call for monitoring methods in which the patient's natural state is less interfered. An ideal device would be non-invasive, minimally restrictive, robust enough to compensate movements of the patients, and would operate without relying on patient's full cooperation.

This paper focuses on the design and development of an unobtrusive vital signs monitoring system particularly suited for long-term monitoring placed under the mattresses. The system is based on the magnetic induction sensing method, designed to infer presence on the bed, breathing and cardiac activity and consists of two coils for excitation and detection. The new detection coil is based on a concentric planar gradiometer for canceling the primary field. The signal acquisition system has been designed using simple electronics to avoid ending up with a complex expensive system. The experimental results were compared with reference signals coming from other known sensors with the different technical basis for benchmarking and identifying the advantages and/or drawbacks of the new system regarding other techniques. The designed system was also studied in regards to safety standards and limitations for the exposure to the magnetic fields. Experimental results confirm the suitability and safety of the sensor for long-term cardiac and respiratory monitoring. The system is able to detect respiration and cardiac activity as well as presence on the bed and changes in position.
\end{abstract}


Keywords: vital sign monitoring, magnetic induction, asymmetric gradiometer, unobtrusive

(Some figures may appear in colour only in the online journal)

\section{Introduction}

Monitoring vital signs while sleeping provides essential information for identifying sleep problems and sleep quality, detection, and treatment of sleep disorders, assessment of different medical conditions or medications' effect on sleep quality and mortality risk assessment associated with sleep patterns in adults and children. An ideal system for sleep monitoring would be non-invasive, minimally restrictive, robust enough to compensate movements of the patients, operates without relying on patient's full cooperation and would not need complex medical environments. Such a system could be of interest for other similar applications where it is not desirable to disturb a subject's physiological and/or emotional state during measurements or in other situations where access to the subject is limited like monitoring neonates, infants at risk of sudden infant health syndrome, burn victims and elderly people.

Assessment of sleep-related disturbances is traditionally performed by polysomnography. While this method provides a rich data set like breathing, heart rate, blood pressure, etc, it can be done only in the clinic environment with the use of wired sensors and skin electrodes. A family of alternative methods is based on measuring small movements of the body due to cardiopulmonary activities, via mechanical coupling. A general term for these methods is ballistocardiography (BCG). Hydraulic under mattress sensors, piezoelectric and piezoresistive transducers, Load cells, strain gauges and fiber-optic pressure sensors are some of the sensors which have been used extensively (Motoi et al 2009, Adami et al 2010, Dziuda et al 2012, Mahdavi et al 2012).

Although BCG has been a successful method among other approaches in unobtrusive monitoring, there are still some challenges to deal with. The measured signal highly depends on the position of the sensor regarding the patient's posture and generally these systems are very sensible to motion artifacts and unwanted signals.

The chest's surface displacements due to respiration and cardiac activity, have been used for monitoring aims in other approaches such as microwave Doppler radar and capacitive ECG. In Doppler radar method, besides having a wireless system to monitor breathing and heart activity, operating at radio microwave frequency bands provides the advantage of low power consumption in these systems. However, the measured signal strongly related to the patients' motions, body orientation and position regarding the antenna (Boric-Lubecke et al 2005, Droitcour et al 2009, Girbau et al 2012). Capacitive ECGs use non-contact electrodes, coupling the signals through a small capacitance but the system can be very susceptible to stray capacitances, interferences and motion artifacts (Chi and Cauwenberghs 2010, Walter et al 2011, Peng and Bocko 2012, Wartzek et al 2012).

Optical and thermal techniques have also been used for non-contact breathing and cardiac activity monitoring. Photoplethysmography, embedding infrared emitting diodes and photo transistors inside mattresses or monitoring thermal signal of the nostril region are some examples of using these techniques (Fei and Pavlidis 2010, Maki et al 2010, Wong et al 2010).

Magnetic induction (MI) could be used as a method for non-contact continuous monitoring of vital signs. Starting from 1960s, the principle has been applied to different medical applications such as measuring resistivity of organs (Tarjan and McFee 1968), tissue characterization (Scharfetter et al 2003), tomography (Griffiths et al 1999, Korjenevsky et al 2000, 


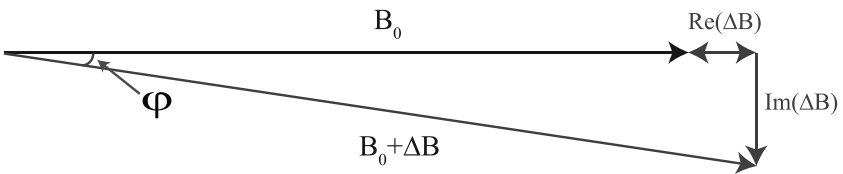

Figure 1. Phasor diagram representing the primary $B_{0}$ and secondary $(\Delta B)$ magnetic fields detected. The total detected field $\left(\Delta B+B_{0}\right)$ lags the primary field by an angle $\Phi$ (Griffiths 2001).

Rosell-Ferrer et al 2006), measurement of liver iron overload (Casañas et al 2004), smart textiles (Teichmann et al 2014), vital signs monitoring (Mahdavi and Rosell-Ferrer 2013), etc.

This paper describes a new low-cost magnetic induction system for monitoring vital signals unobtrusively and introduces a new magnetic planar gradiometer for signal detection. The hardware and the coil configuration are designed specifically for detection of small perturbations due to physiological activities. In addition, system's safety in regards to the patient being exposed to the magnetic field, is studied and preliminary results on volunteers are presented in this paper.

\section{Magnetic induction; measurement basis}

Based on Maxwell theory, when a conductive object is placed in a time varying magnetic field $\left(B_{0}\right)$, eddy currents are induced in the object. These currents produce a secondary magnetic field (perturbations, $\Delta B$ ) that can be detected by a properly designed receiver system.

From Griffiths et al (1999) and Korjenevsky and Cherepenin (1999) for a sample of material between an excitation coil and a detection coil we have $\Delta B / B_{0} \propto \omega\left(\omega \varepsilon_{0} \varepsilon_{r}-j \sigma\right)$ where $\sigma$ is the conductivity of the sample, $\varepsilon_{r}$ is its relative permittivity, $\varepsilon_{0}$ is the permittivity of free space, $\omega$ is the angular frequency of the excitation and $j=\sqrt{-1}$. Thus, the conduction currents induced in the sample cause a component of $\Delta B$ which is proportional to frequency and lags the primary field, $B_{0}$, by $90^{\circ}$. In general, $\Delta B$ will have real and imaginary components representing the permittivity and conductivity of the sample respectively (Griffiths 2001) (figure 1). For biological tissues, the conductivity (imaginary) component will normally be dominant (Griffiths 2001). As a general challenge, in biomedical applications, with tissue conductivity in the range between $0.02 \mathrm{~S} / \mathrm{m}$ (bone cortical) and $2 \mathrm{~S} / \mathrm{m}$ (cerebrospinal fluid), the $\left|\Delta B / B_{0}\right|$ is generally $\ll 1$ (Griffiths 2001) and increases in proportion to the excitation frequency.

The $\Delta B$ could be measured via one or an array of detection coils. In the single coil method, the same coil that has worked as excitation is used for detecting the signal. For example, the coil could be used as a part of a Colpitts oscillator, self oscillating with a frequency between 4 to $10 \mathrm{MHz}$. The induced secondary field is measured indirectly by the effective impedance change produced by it (Teichmann et al 2010) through the changes which were produced in the oscillation frequency. However, developed systems by this technique suffer from several artifacts. Moving an object relatively to the coil poses a change in the conductivity distribution of the region measured by the sensor while on the other hand, parasitic stray capacitance changes the oscillation frequency.

Using separate coils for excitation and detection has been investigated in various measurement systems designed for vital signs monitoring and tomography. The secondary induced field in this method is directly measured by a (or a set of) receiver coil(s). Relative voltage changes $\Delta V / V_{0}$ is acquired from the receive(s), $\Delta V$ is the voltage change and $V_{0}$ is the 
induced voltage in one receiver coil in the absence of a perturbation. Considering the small secondary signal and the large background signal, the design process especially the coil's configuration is very critical in the overall performance of the MI systems. Digital processing of such signals requires an impractically high dynamic range of the ADCs. Therefore, it is recommended (Scharfetter et al 2005) to reduce the induced voltage $V_{0}$ in the unloaded system while preserving maximum sensitivity to conductivity changes.

The three possible cancellation methods are:

- producing zero magnetic flux in the unperturbed situation by orientation of the receiver coil (zero flux coil, ZFC) (Watson et al 2004)

- subtraction of the signals in a pair of differential coils (coaxial and planar gradiometer)

- electronic subtraction of $V_{0}$ by adding $-V_{0}$ from a controllable signal generator

Since the first and second methods are easier to implement and more important they cancel the primary field (voltage) at an earlier stage, they are preferable regarding the second method. Comparing these two methods, in the case of ZFC, no voltage is induced by the primary field. This is an advantage over the gradiometer structure because the subtraction of two relatively high voltages in gradiometer coils is never perfect leaving a residual voltage and causes some drifts. On the other hand, a disadvantage of ZFC is the higher susceptibility to interferences from far RF sources (Scharfetter et al 2005) which in the gradiometer such interferences are canceled up to a rather high degree.

The coaxial type of gradiometer and the ZFC are not suitable for being placed under the bed or mattress. While the planar gradiometer seems a suitable option for our application, it shows a zero sensitivity plane in the center which is totally undesirable. Our new approach was to design an concentric planar gradiometer $(\mathrm{CPG})$ in order to address the mentioned challenges. However, the CPG is susceptible to interferences from far RF sources.

\section{Materials and methods}

\subsection{Mathematical models based on finite elements}

Computer modeling of electromagnetic fields distribution in biological tissues is important to estimate and evaluate expected signals in experimental setup and real measurements. Distribution and intensity of the field lines and flux density and sensitivity of the system to various parameters are among the simulated cases. COMSOL Multiphysics has been used for simulations applying the finite element method (FEM).

3.1.1. Thorax modeling in COMSOL. For simplicity reasons and to avoid complicated meshing problems in FEM simulation, a simplified anatomical 3D model of the human trunk was designed within COMSOL. The developed model consists of a sphere as the surrounding area, a cylinder for trunk structure and cones and spheres for lungs and heart. A current carrying coil is located in front of the chest as the excitation coil. Figure 2 illustrates the adjustment of our model to a computerized tomography (CT) scan of thorax and in figure 3 the final 3D model used for simulations is shown.

The excitation and detection coils were simulated as edges instead of toroids in order to avoid the necessity of small elements generation inside the coil domains and excitation current was applied to the coil as a boundary condition. The total magnetic flux due to induced magnetic field passing through the detection coil is calculated by the surface integration of the orthogonal (to the surface) component of the magnetic field in the detection coil. Since in magnetic induction, it is believed that the magnetic fields are not blocked by poorly conducting 


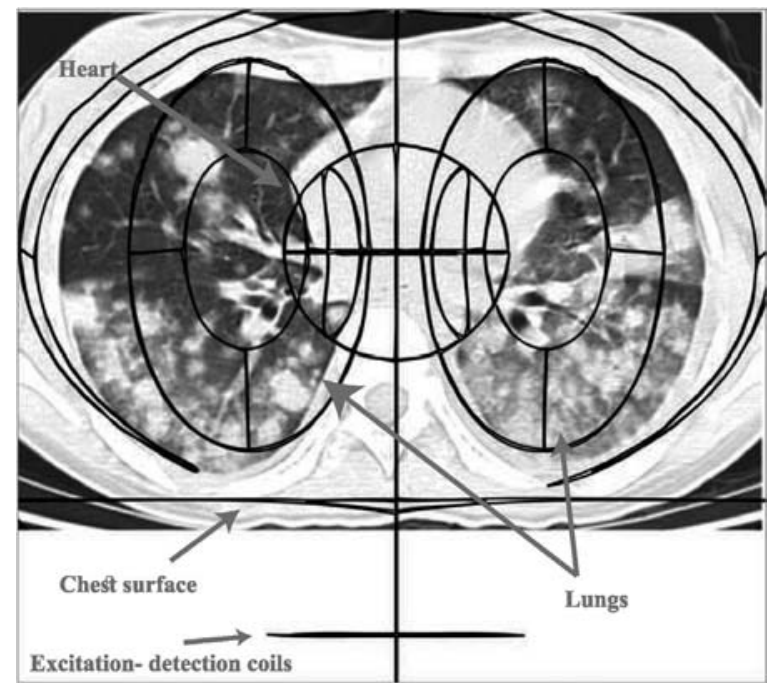

Figure 2. 3D model's adjustment over a CT of thorax.

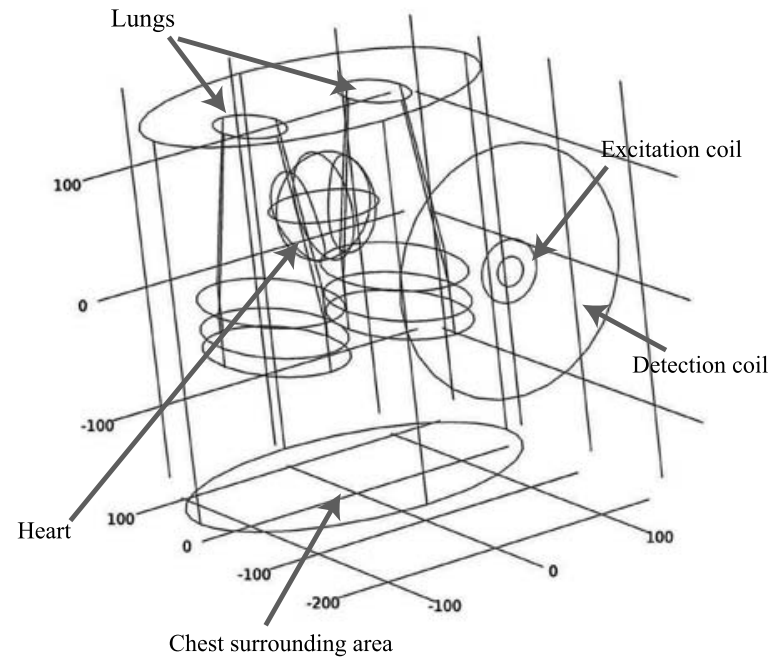

Figure 3. The final 3D model.

tissues such as bones (Griffiths et al 1999), the model does not contain ribs or other poor conductive parts of the thorax to reduce the complexity of the resulted mesh. The dimensions and volumes of the designed organs are approximately the ones for a healthy normal male. Main tissue types used as material in the simulation, dielectric properties of the tissues at $10 \mathrm{MHz}$ and number of finite elements of each organ are shown in table 1. The applied passive electrical properties of body tissues were derived from Gabriel et al (1996). The application mode chosen was Magnetic Fields (mf) in COMSOL's AC/DC module.

3.1.2. Exposure to electromagnetic field: limitations and standards. Magnetic induction monitoring systems are based on the exposure of electromagnetic fields, so the limitations and standards for the exposure to these fields should be considered in the design process. Exposure 
Table 1. Number of elements and dielectric properties at $10 \mathrm{MHz}$.

\begin{tabular}{lcll}
\hline Tissue & Number of elements & Conductivity S/m & Relative permittivity \\
\hline Muscle & 30586 & 0,6 & 170 \\
Heart & 2847 & 0,4 & 293 \\
Lungs inflated & 7484 & 0,22 & 123 \\
Lungs deflated & 7484 & 0,44 & 180 \\
\hline
\end{tabular}

to time-varying electromagnetic field results in internal body currents and energy absorption in tissues. The quantity of energy absorption and level of internal currents depend on the coupling mechanisms and the frequency involved.

Specific absorption ratio (SAR) is a measure of the rate at which energy is absorbed by the body when exposed to a radio frequency (RF) electromagnetic field; It is defined as the power absorbed per mass of tissue and has units of watts per kilogram $\left(\mathrm{W} \mathrm{kg}^{-1}\right)$.

The most commonly used safety standards are the ANSI/IEEE C95.1 (Safety 2005) and ICNIRP (International Commission on Non-Ionizing Radiation 1998). In accordance with ICNIRP recommendations, the maximum field strength is limited by the SAR which shall not exceed $2 \mathrm{~W} \mathrm{Kg}^{-1}$ for head and torso. As a safety requirement, the estimated SAR of the developed sensor system was simulated and studied.

For simulating the SAR, parametric sweep study mode has been used to find the solution. Frequency and the distance between the excitation coil and the body were defined as variables to study their impact on SAR and determine a safe margin of distance and frequency for the system. Equation (1) was used to calculate SAR. In this equation, $m f . Q H$ is the electromagnetic heating averaged over the density of the tissue. Both inflated and deflated lungs were considered and simulated.

$$
\mathrm{SAR}=m f \cdot Q h / \text { rho }(\text { trunk })
$$

\subsection{MI system design and implementation.}

As stated previously (section 2), we have used the planar gradiometer approach as a primary field cancellation method. Different topologies were studied and tested and the final design is explained here. The designed system is based on a coil as excitation, a detection coils and a phase sensitive detector. The detection coil is a concentric planar gradiometer (CPG) which will be explained later in this section. The excitation signal is provided by a signal generator at a frequency of $10.7 \mathrm{MHz}$.

3.2.1. Excitation coil. The excitation coil is an 8-turn spiral coil with an inner diameter of $5 \mathrm{~cm}$ implemented on PCB. Based on the modified Wheeler formula (Wheeler 1928, Mohan et al 1999), the self-inductance of the excitation coil is $7 \mu \mathrm{H}$. That is for an 8 turn spiral with a wire diameter of $0.6 \mathrm{~mm}$, turn spacing of $0.8 \mathrm{~mm}$ and an inner diameter of $50.8 \mathrm{~mm}$. The measured self-inductance of the coil (with HP4192A impedance analyzer) however, is $8.8 \mu \mathrm{H}$. The difference comes from the equation's approximations and layout dependent coefficients.

3.2.2. Detection coil, $C P G$. The $\mathrm{CPG}$ is a one-turn antenna with a total measured inductance of $1 \mu \mathrm{H}$. Similar to the excitation coil, the CPG is implemented on PCB, located symmetrically to the excitation coil. Through simulations, cancellation ratio and the optimum radius 


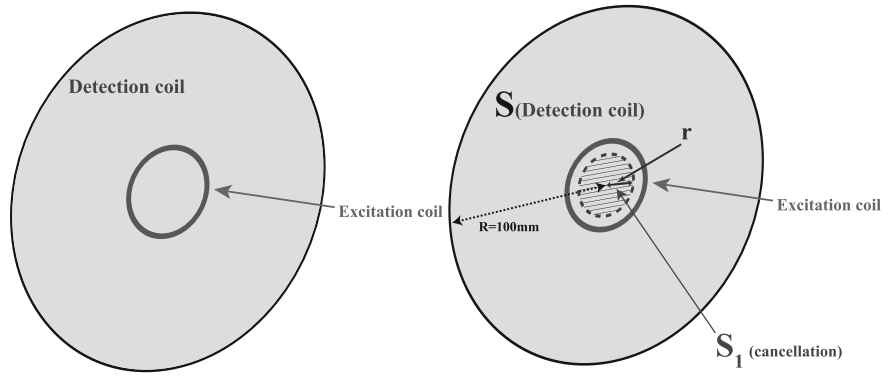

(a)

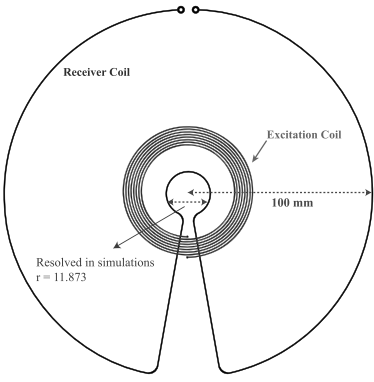

(c)

Figure 4. Coil design; (a) detection coil with no cancellation, (b) detection coil with cancellation, (c) final implemented excitation-detection coil structure.

Table 2. CPG Simulation's assumptions.

\begin{tabular}{llcc}
\hline Frequency & & 10 & $\mathrm{MHz}$ \\
\hline Object's distance from the sensors & & 50 & $\mathrm{~mm}$ \\
\hline Object conductivity & Lungs & 0,44 & $\mathrm{~S} / \mathrm{m}$ \\
& Heart & 0,5 & $\mathrm{~S} / \mathrm{m}$ \\
& Muscles & 0,6 & $\mathrm{~S} / \mathrm{m}$ \\
\hline Exc coil radius & & 25 & $\mathrm{~mm}$ \\
\hline Det outer radius & & 100 & $\mathrm{~mm}$ \\
\hline
\end{tabular}

for CPG were calculated to ensure and validate the design. Two set of simulations were performed in order to calculate the cancellation ratio (at free space and in the presence of the object, with and without cancellation). In addition, parametric solver was used to resolving the CPG's inner section radius where the cancellation is at maximum. The object is the body model with the conductivities mentioned in table 1 . The conductivity applied to the lungs is for the deflated mode.

The first simulation was performed with a one turn excitation coil and a one turn simple detection coil (without any cancellation surface), placed concentrically. The separation between two coil plates is $2 \mathrm{~mm}$ and the outer section's radius of CPG is $100 \mathrm{~mm}$ (see figure 4(c)). The simulation was then repeated with the same excitation coil but a different receiver. The new detection coil, has a second surface $\left(S_{1}\right.$, see figure $\left.4(\mathrm{~b})\right)$ acting like a second coil which is connected in series to the first one. Based on the gradiometer's principle, the excitation field in the first and second detection coils is cancelled by currents induced in the first and second detection surfaces adding up to zero:

$$
\int_{S} \int \vec{B}_{0} \cdot \mathrm{d} \vec{s}-\int_{S_{1}} \int \vec{B}_{0} \cdot \mathrm{d} \vec{s}=0
$$

Table 2 shows the assumptions and specifications of the simulation.

As explained before, the effect of primary magnetic field $\left(B_{0}\right)$ appears in the real component of the detected signal. Comparing the results of CPG simulation with the case of a simple coil for detection, a cancellation factor of 5 orders of magnitude in the background signal could be observed. On the contrary, the imaginary part of the signal (see table 3 ) which is the contribution of the eddy currents, experiences fewer changes after cancellation and stays at the same level of magnitude. The residual real part remained after applying the cancellation is the contribution of object's permittivity. 
Table 3. CPG simulation results.

\begin{tabular}{lllc}
\hline Radius & $\begin{array}{l}\text { Simulation } \\
\text { description }\end{array}$ & $\begin{array}{l}\text { Measured } \\
\text { flux }(\mathrm{Wb})\end{array}$ & $\begin{array}{l}\text { Measured } \\
\text { voltage }(\mathrm{V})\end{array}$ \\
\hline $\mathrm{R}=100 \mathrm{~mm}, \mathrm{r}=\mathrm{NA}$ & $\begin{array}{l}\text { No cancellation, } \\
\text { no object }\end{array}$ & $121 E^{-10}+0 i$ & $0.76+0 i$ \\
$\mathrm{R}=100 \mathrm{~mm}, \mathrm{r}=\mathrm{NA}$ & $\begin{array}{l}\text { No cancellation, } \\
\text { with object }\end{array}$ & $123 E^{-10}-8.83 E^{-11} i$ & $0.77-0.005 i$ \\
$\mathrm{R}=100 \mathrm{~mm}, \mathrm{r}=11.8739 \mathrm{~mm}$ & $\begin{array}{l}\text { CPG, no object } \\
\mathrm{R}=100 \mathrm{~mm}, \mathrm{r}=11.8739 \mathrm{~mm}\end{array}$ & $0.01 E^{-10}+0 i$ & $9.25 E^{-06}+0 i$ \\
\hline
\end{tabular}

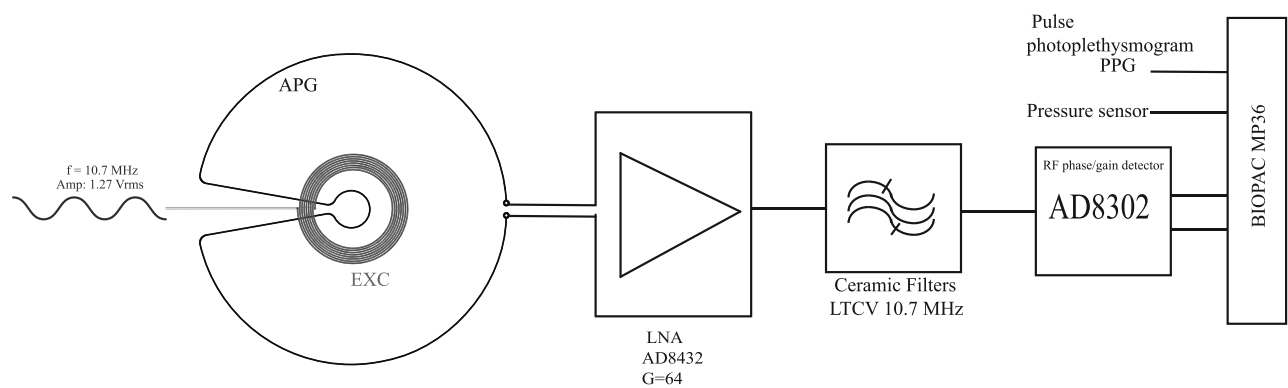

Figure 5. MIS system block diagram plus BIOPAC for acquiring reference signals

The results from simulations face some practical limitations in the implementation phase. The connections and the capacitive coupling between wire lines are some of the important bottlenecks which have to be considered in the hardware implementation. The final implemented radius for the inner section of the $\mathrm{CPG}$ is $11.7 \mathrm{~mm}$ and the measured inductance of the final PCB implementation of CPG is $1 \mu \mathrm{H}$.

3.2.3. Acquisition system. Given the small signals received by CPG, achieving the required phase measurement precision for vital signs monitoring application is a major challenge. Thus the signal amplification and phase sensitive acquisition system designs are crucial for the performance of the system.

In our design, the signal received at CPG, will be amplified by a two stage low noise amplifier (AD8432) with a gain of 64 and an input voltage noise of $0.85 \mathrm{nV} \sqrt{\mathrm{Hz}}^{-1}$. The amplified signal is then filtered by ceramic filters at $10.7 \mathrm{MHz}$ before being introduced to a gain and phase detector. AD8302, an RF/IF gain-phase detector with an accurate phase measurement scaling of $10 \mathrm{mV} \mathrm{deg}^{-1}$, is used for demodulation and phase detection. The excitation signal is provided by a signal generator at an operating frequency of $10.7 \mathrm{MHz}$. Figure 5 shows the block diagram of the developed system. As a reference, for breathing and cardiac activity signals, Pulse plethysmogram (PPG) and the pressure transducer of a BIOPAC-MP36 system have been used to monitor and compare the signals detected by MIS with mechanical-based system results.

\subsection{Description of experiment setup and protocols}

The experimental setup for the verification of the approach consists of the coils, the amplification and detection circuits and BIOPAC MP36. For experiments with volunteers, pulse plethysmogram (PPG) sensor and pressure transducer of the BIOPAC-MP36 are also used. The 


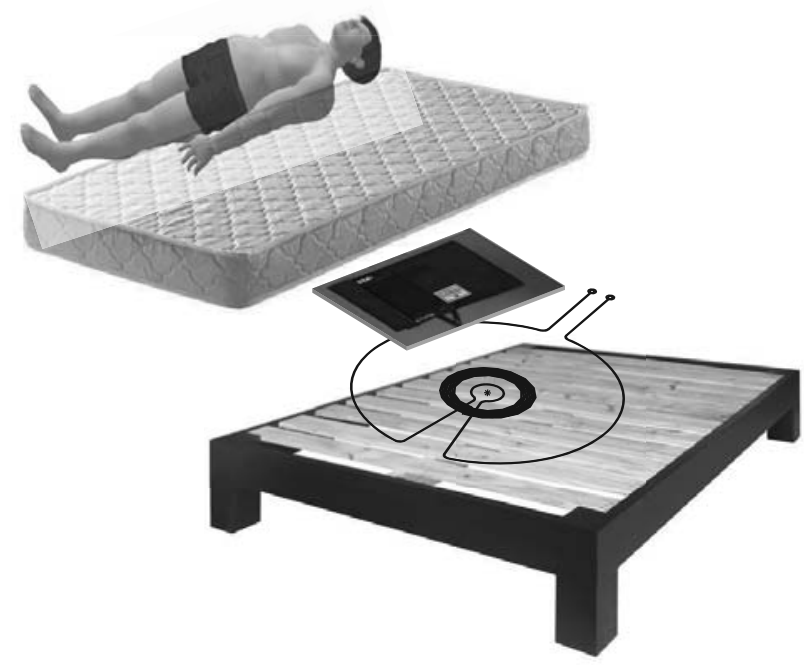

Figure 6. Volunteer's position in regards to MI system.

complete block diagram of the system is shown in figure 5. The coils are placed symmetrically with a $2 \mathrm{~mm}$ distance from each other. The distance between the coils and the object under test/the volunteer is $5 \mathrm{~cm}$. The vital signs monitoring experiments took $100 \mathrm{~s}$ and the sampling frequency was 500 sample per second. No metallic part or wire was used close/orthogonal to the field lines. The bed base and the mattress are all made with plastic, foam or wood in order to avoid any systematic error due to moving/presence of well-conducting and/or ferromagnetic material near the object space.

3.3.1. Signal linearity with conductivity changes. To study the system's behaviour measuring different conductivities, six different conductivities were obtained by adding sodium chloride to a one-litre bottle of distilled water. The bottle was fixed at a distance of $5 \mathrm{~cm}$ from the sensor's head. The experiment was simulated in COMSOL in order to be used as a reference for comparison.

3.3.2. Vital signs detection experiments. In these experiments, the distance between sensor's head and the volunteer was $5 \mathrm{~cm}$ (see figure 6). The test procedure started with breathing normally, followed by a period of apnea and later normal breathing again. Experiments were done in supine and prone positions with an adult volunteer.

\section{Results and discussions}

\section{1. $S A R$ calculations}

As mentioned before, the estimated SAR of the system was studied through simulations to assure the safety of the system. The SAR was calculated for a range of frequencies from $100 \mathrm{KHz}$ to $10 \mathrm{MHz}$ and a distance-between the object/body and the coils_-of $5 \mathrm{~cm}$ to $20 \mathrm{~cm}$.

The estimation of the absorption rate at the mentioned frequencies and distances shows that even considering the worst case for exposure to the magnetic field (a frequency of $10 \mathrm{MHz}$ and 
$5 \mathrm{~cm}$ distance), the maximum SAR is lower than the safety standards' limits. That is, for an excitation current of $1 \mathrm{~A}$ in a one turn coil at a distance of $5 \mathrm{~cm}$ from the chest at a frequency of $10 \mathrm{MHz}$, we are more than one order of magnitude under the safety limits imposed by ICNIRP standard (maximum calculated SAR $=0.0056 \mathrm{~W} \mathrm{Kg}^{-1}$ ).

\section{2. $C P G$ simulation results}

The optimum radius obtained by simulations for the cancellation radius is $11.87 \mathrm{~mm}$. Figure 4 and table 3 show the different scenarios and the calculated flux in those scenarios of the simulation. The table shows the measured secondary signal;

- in the absence of the object and when no cancellation technique was applied to the detection coil

- in the presence of the object and when no cancellation technique was applied to the detection coil

- in the absence of the object and when CPG has been used

- in the presence of the object and when CPG has been used

Another interesting possibility for the coil structure, could be the use of the planar gradiometer with zero sensitivity plane in the centre (explained before). Mounting this gradiometer in the mattress in a way that it wouldn't intersect with the thorax, would solve the zero sensitivity plane problem and has the benefit of immunity to the far sources of interference. However, to the authors, this structure will have one limitation: it has an intrinsic ambiguity in the sign (increase or decrease) and the source (thorax or abdomen) of the detected secondary field as the two coils are connected in opposition. On the contrary, with the CPG, it is possible to know if the secondary field increases or decreases in the region in front of the concentric coils. This allows putting together a matrix of sensors in the mattress to increase the sensitivity of the system.

\subsection{System's sensitivity to conductivity changes}

The results of the experiments described in section 3.3.1 - the measured real and imaginary part of the received signals (due to changes in conductivity) - are shown in figure 7 together with the theoretical calculations and simulation results.

The linearity of the received signal with changes in conductivity can be seen in the experimental results and it is in accordance with the simulation and theoretical estimations (based on equation (3)). The practical maximum cancellation ratio obtained in the experiments (through adjusting the coils' position) was measured as 150 times. To obtain the same real part in the experiments as in the theory/simulations a cancellation factor of $10^{5}$ is necessary.

$$
\Delta B=k \omega(-j \sigma+\omega \varepsilon)
$$

In an ideal MI system, the coupling between the object and the coils is expected to be only by the magnetic field. However, in a practical system, the electric field (capacitive) coupling also exists. The general impact of capacitive coupling and different coupling scenarios are explained in Goss et al (2002) and Mahdavi and Rosell-Ferrer (2013).

The results for the real part of the signal (shown in figure 7) is not zero because of:

- cancellation of the primary field is not ideal so a part of this signal is due to the residual primary background signal 


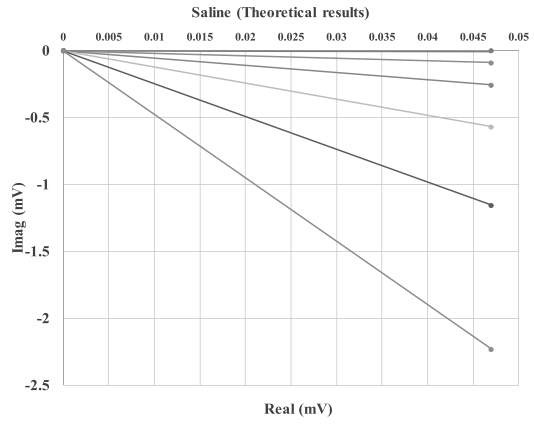

(a)

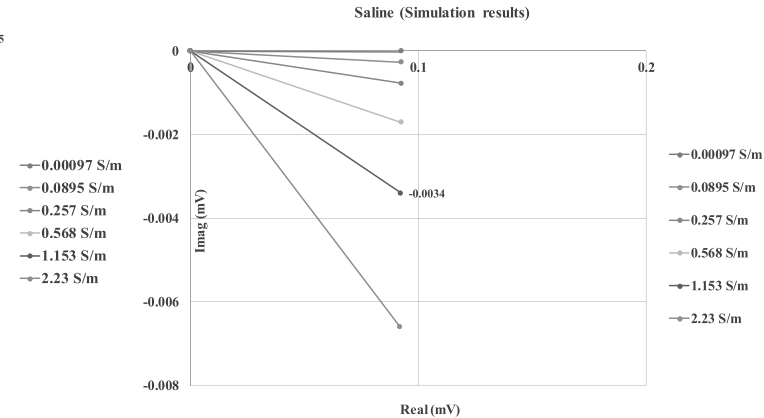

(b)

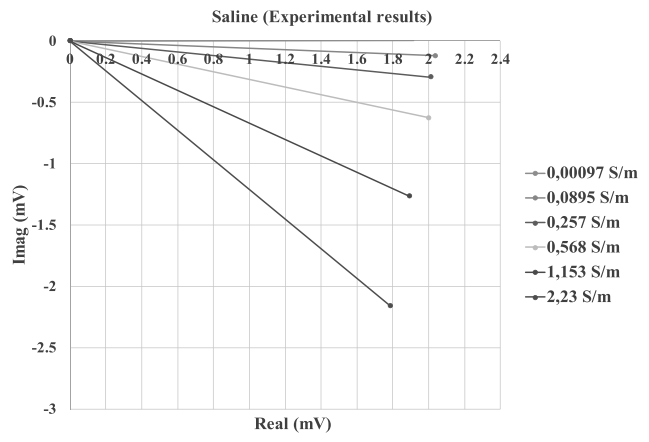

(c)

Figure 7. Measured signal, real and imaginary components for different conductivities of saline solution; (a) theoretical results, (b) simulation results, (c) experimental results.

- the real part due to the permittivity of the tissues (equation (3)), that is orders of magnitude lower than the residual signal from the primary field

- the electric field coupling (capacitive coupling)

In order to suppress the capacitive coupling we have tried experimentally a comb screen in this coil structure and also in previous planar gradiometers. In our experiments, comb screening did not cause a significant change in the detected real part. This could be because of the relative poor cancellation factors that could be obtained in practice, between 100 and 500 . In addition, the use of screens increases the capacitive coupling between the coils and the ground, introducing an additional phase shift in the detected signals.

The residual primary field signal after cancellation should be in-phase with the primary field but due to the capacitive coupling, the detected field could have any random phase. The capacitive component sums up with the contribution of eddy currents (in the object) and the resulted phase would be from a rotated signal.

\subsection{Vital signs monitoring}

Figures 8 and 9 show the measurements of vital signs registering in supine and prone positions respectively. The signals shown in these figures are directly obtained from BIOPAC MP36 system and not further processed. The applied digital filter from BIOPAC is a high pass filter with cut off frequency of $0.05 \mathrm{~Hz}$. 

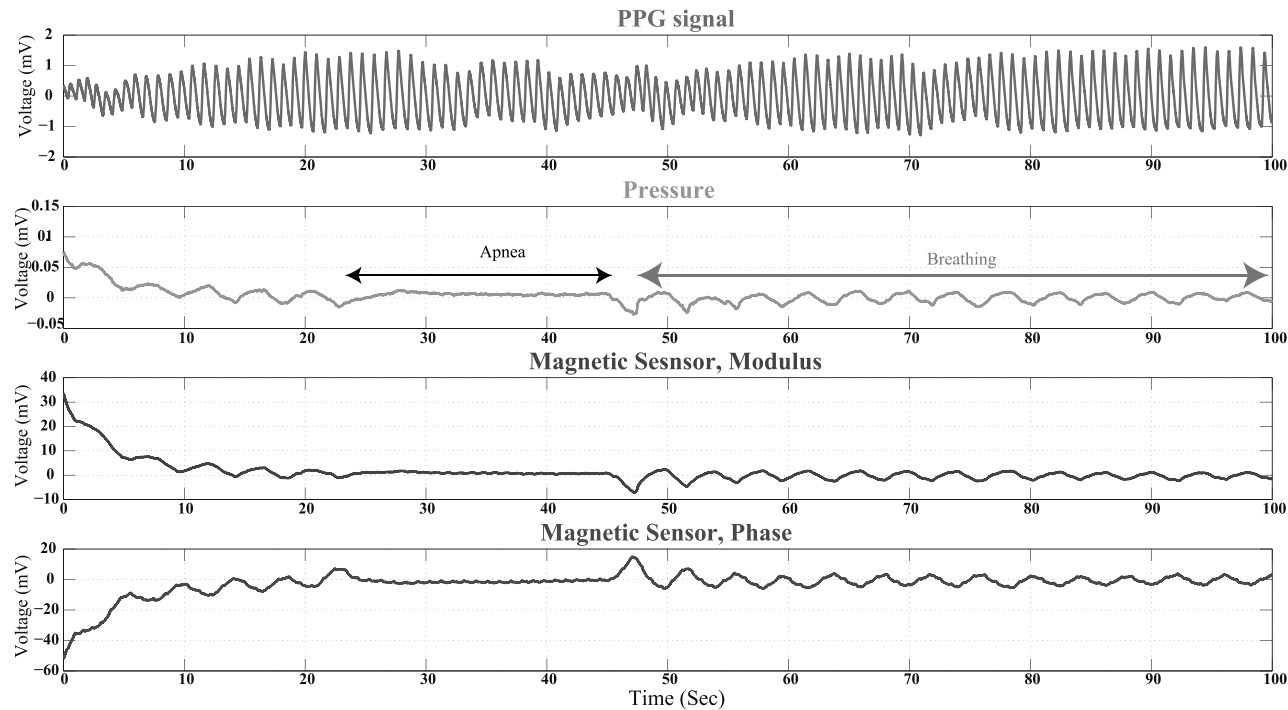

Figure 8. Vital signs monitoring, supine position ( $5 \mathrm{~cm}$ separation).
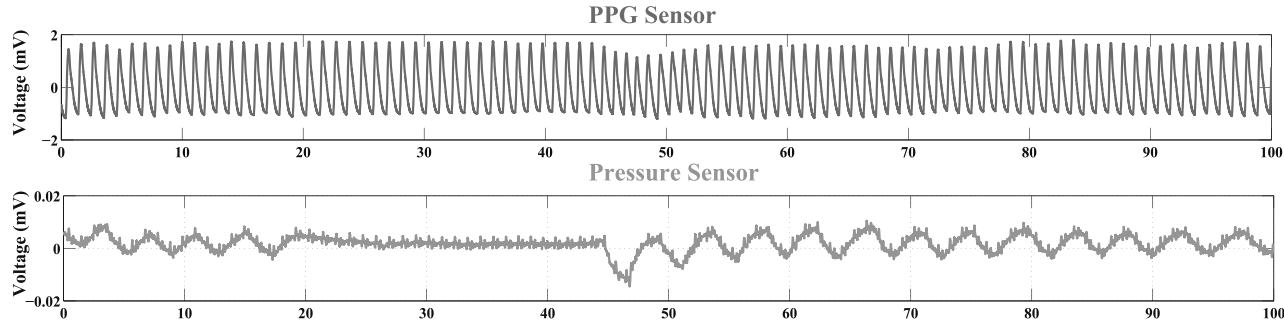

Magnetic Sesnsor, Modulus

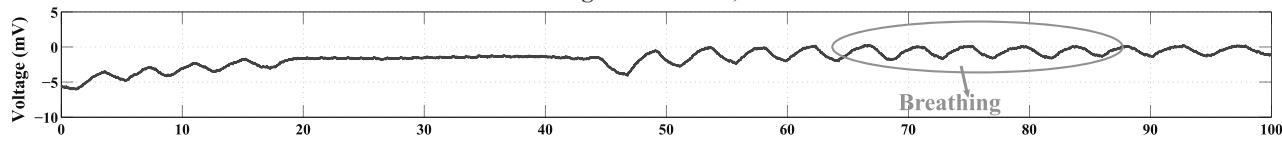

Magnetic Sensor, Phase

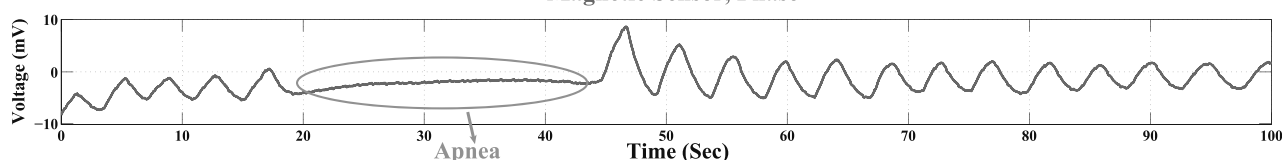

Figure 9. Vital signs monitoring, prone position ( $5 \mathrm{~cm}$ separation).

Figures 10 and 11 gives a comparison insight of the cardiac activity at apnea period of prone and supine position, for all four sensors. The pressure signal has been enlarged for demonstration since the received signal from this sensor is very small.

As it could be seen, monitoring breathing signal is not an issue and both MI and pressure sensors can detect it. In MI simulations (with a $5 \mathrm{~cm}$ distance between the body and the coils), changing the conductivity and volume of the lungs from deflated to inflated mode, cause a 6.8 percent decrease in the imaginary component of the signal while the real component grows about 0.19 percent. However, inhalation of air into the lungs produce a small increase in the thorax volume which could be considered as a small movement toward the sensors. An 


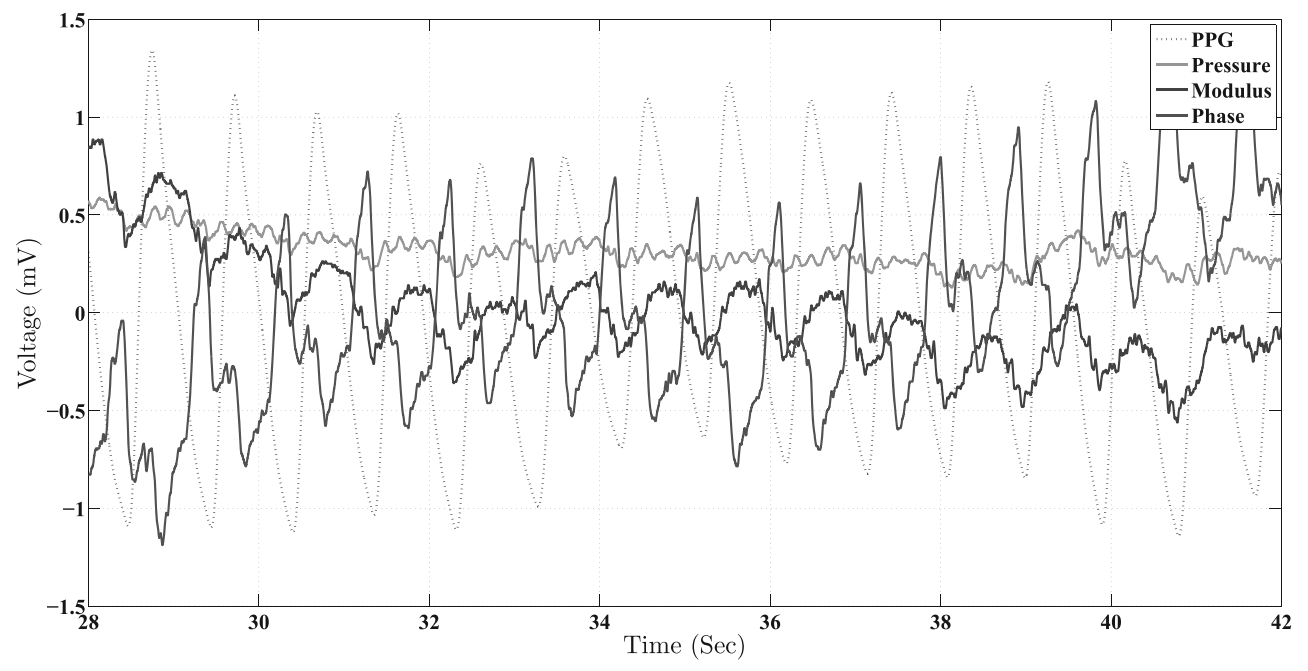

Figure 10. Apnea signal in Supine position, note that the signal from pressure sensor has been scaled 50 times for demonstration reasons.

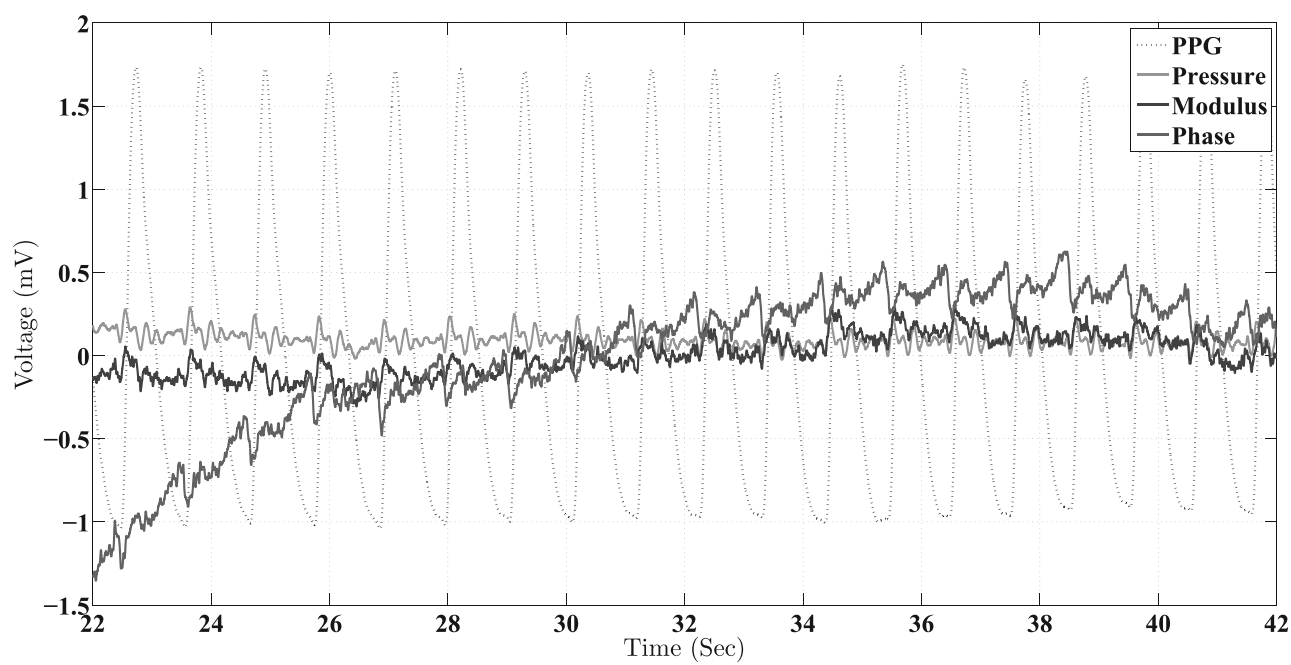

Figure 11. Apnea signal in Prone position, note that the signal from pressure sensor has been scaled 50 times for demonstration reasons.

increase of 0.5 litre in thorax's volume, results in an approximate $3 \mathrm{~mm}$ displacement of the body toward the coils. This modification in distance affects both components of the received signal. The imaginary component, in this case, decreases 0.21 percent changing from deflation to inflation mode while the increase in the real part of the signal is 4.3 percent. The experiments results' are in accordance with the results from simulations. As shown in figure 8, when the lungs are filled with air (inflation mode) the modulus of the magnetic signal increases while the phase decreases. The decrease in phase signal is due to decrement of conductivity of the lungs while the increment in the modulus could be caused by the chest's expansion which slightly changes the distance between the sensors and the body, and also modify the distribution of the currents in the surface. 
A change in the surface geometry of the object or the patient, close to the coils, will introduce a change in the capacitive coupling and also in the received secondary magnetic field. Both cases will produce a change relative to the posture of the patient or body movements related to breathing or cardiac activity. The advantage of the magnetic system is that if there is no movement, the magnetic field that penetrates the body still produces a secondary signal that could be detected. On suppressing the capacitive coupling, based on our objectives-to make a simple yet robust system with simple electronics-we try to make use of the capacitive coupling since it could provide us with more information and not suppressing it entirely.

Comparing the signals of supine and prone positions indicates that the signal from prone position contains more information in regards to cardiac activity due to its position advantage. In prone position, the sensors are closer to the heart, to the blood volume changes in heart's major vessels (Aorta) and to the lung perfusion while in the supine position, the distance is higher and other structures like vertebral column are located in between. However, both supine and prone positions show more reliable signals than the pressure sensor's outcomes. Lateral recumbent positions are other two positions which were measured for several volunteers in addition to the supine and prone positions. Although the variability of the signals in these two lateral positions were relatively high, the preliminary conclusions of the authors is that the left side position (lying over the left shoulder) shows higher sensitivity to cardiac activity while for breathing, no big difference has been identified. The experiments also showed that volunteers' breathing habits affects the received signals. It has been observed that breathing through stomach (belly breathing) causes bigger periodic body movements and can have impacts on the detected signal by modifying the distance between the body and the sensor and changing current distributions.

The distance between the body and the sensors in the described experiments was $5 \mathrm{~cm}$. In determination of this distance several factors were considered, simulated and experimentally tried. The main driving factor is the attenuation of the magnetic field due to distance increment. Another reason was the practicality of the system implementation. Bed bases and mattresses have different sizes and that would directly affect the system's ability to detect signals. Distances of 20, 10 and $5 \mathrm{~cm}$ were experimented and for this system with the described structure and characteristics, $5 \mathrm{~cm}$ is found to be a proper separation between sensor head and the body. In case of thick mattresses, this separation could be obtained by embedding the sensor inside the mattress.

Spatial coverage of the system for reliable detection was both simulated and measured experimentally. Assuming that the surface of the bed is on XY plane, the described system has a reliable coverage of about $10 \mathrm{~cm}$ for the displacements along $\pm X$. For the displacements along the $Y$ axis, the coverage is higher but then the detected signal in different locations along $Y$ axis could be a contribution of lungs, heart and also other abdominal body organs.

\section{Conclusions}

A new non-contact magnetic induction sensor system for monitoring vital signs has been developed and described in this paper. As explained in detail, the system is a low-cost, non-contact planar system designed to be placed under the bed or mattress for unobtrusive monitoring applications such as sleep monitoring, neonates monitoring, etc. The detection is a concentric planar gradiometer which has been optimized to minimize the impact of the primary magnetic field in the measured secondary signal.

System's safety study indicates that the developed system is safe to be used for continuous monitoring of breathing and cardiac activity for patients in terms of being exposed to 
magnetic fields. The results show that the system placed at $5 \mathrm{~cm}$ of the patient's chest, can detect the presence of the patient on the bed, breathing and also cardiac activity. The signal levels decrease with the increment of the distance. Considering the bed to be on XY plane, a practical coverage of $10 \mathrm{~cm}$ along $\pm X$ axis was obtained for reliable signal measurement.

MI phase signal shows more sensitivity than MI magnitude signal. Breathing causes a movement in patient's back which modifies its distance from sensors. That is why the measured breathing signal could be a combined signal from magnetic coupling and electric field coupling.

Comparing signals from pressure and MI sensors point out that in some cases/positions the cardiac signal is better detected in either of signals. As stated, the source for the received signal could be a combination of thorax or/and abdomen changes — which may cause changes in electric field coupling or surface eddy currents-and the internal eddy currents produced by conductivity changes originated from lung perfusion, muscle perfusion or blood volume changes in heart and major vessels.

Adami A M, Pavel M, Hayes T L and Singer C M 2010 Detection of movement in bed using unobtrusive load cell sensors IEEE Trans. Inf. Technol. Biomed. 14 481-90

Boric-Lubecke O, Lubecke V, Host-Madsen A, Samardzija D and Cheung K 2005 Doppler radar sensing of multiple subjects in single and multiple antenna systems 7th Int. Conf. on Telecommunication in Modern Satellite, Cable and Broadcasting Services vol 1 pp 7-11 (IEEE)

Casañas R, Scharfetter H, Altes A, Remacha A, Sarda P, Sierra J, Merwa R, Hollaus K and RosellFerrer J 2004 Measurement of liver iron overload by magnetic induction using a planar gradiometer: preliminary human results Physiol. Meas. 25 315-23

Chi Y M and Cauwenberghs G 2010 Wireless non-contact EEG/ECG electrodes for body sensor networks 2010 Int. Conf. on Body Sensor Networks pp 297-301

Droitcour A, Boric-Lubecke O and Kovacs G 2009 Signal-to-noise ratio in doppler radar system for heart and respiratory rate measurements IEEE Trans. Microw. Theory Tech. 57 2498-507

Dziuda L, Skibniewski F W, Krej M and Lewandowski J 2012 Monitoring respiration and cardiac activity using fiber Bragg grating-based sensor IEEE Trans. Biomed. Eng. 59 1934-42

Fei J and Pavlidis I 2010 Thermistor at a distance: unobtrusive measurement of breathing IEEE Trans. Biomed. Eng. 57 988-98

Gabriel C, Gabriel S and Corthout E 1996 The dielectric properties of biological tissues: I. Literature survey Phys. Med. Biol. 41 2231-49

Girbau D, Lázaro A, Ramos Á and Villarino R 2012 Remote sensing of vital signs using a doppler radar and diversity to overcome null detection IEEE Sens. J. 12 512-8

Goss D, Mackin R, Crescenzo E, Tapp H and Peyton A 2002 Understanding the coupling mechanisms in high frequency EMT 3rd World Congress on Industrial Process Tomography (Banff) p 364/369

Griffiths H 2001 Magnetic induction tomography Meas. Sci. Technol. 12 1126-31

Griffiths H, Stewart W and Gough W 1999 Magnetic induction tomography: a measuring system for biological tissues Ann. New York Acad. Sci.

International Commission on Non-Ionizing Radiation 1998 ICNIRP guidelines for limiting exposure to time-varying electric, magnetic and electromagnetic fields (up to $300 \mathrm{GHz}$ ) Health Phys. 74 494-522

Korjenevsky A V and Cherepenin V A 1999 Progress in realization of magnetic induction tomography Ann. New York Acad. Sci. 873 346-52

Korjenevsky A, Cherepenin V and Sapetsky S 2000 Magnetic induction tomography: experimental realization Physiol. Meas. 21 89-94

Mahdavi H, Ramos-Castro J, Giovinazzo G, García-González M A and Rosell-Ferrer J 2012 A wireless under-matress sensor system for sleep monitoring in people with major depression The Ninth IASTED Int. Conf. on Biomedical Engineering

Mahdavi H and Rosell-Ferrer J 2013 A magnetic induction measurement system for adult vital sign monitoring : evaluation of capacitive and inductive effects $X V$ Int. Conf. on Electrical Bio-Impedance \& XIV Conf. on Electrical Impedance Tomography vol 012085 
Maki H, Ogawa H, Tsukamoto S, Yonezawa Y and Caldwell W M 2010 A system for monitoring cardiac vibration, respiration and body movement in bed using an infrared 32nd Annual Int. Conf. of the IEEE Engineering in Medicine and Biology Society pp 5197-200

Mohan S S, Hershenson M D M, Boyd S P and Lee T H 1999 Simple accurate expressions for planar spiral inductances IEEE J. Solid-State Circuits 34 1419-20

Motoi K et al 2009 A fully automated health-care monitoring at home without attachment of any biological sensors and its clinical evaluation 31st Annual Int. Conf. of the IEEE Engineering in Medicine and Biology Society pp 4323-6

Peng G and Bocko M F 2012 A low noise, non-contact capacitive cardiac sensor Annual Int. Conf. of the IEEE Engineering in Medicine and Biology Society pp 4994-7

Rosell-Ferrer J, Merwa R, Brunner P and Scharfetter H 2006 A multifrequency magnetic induction tomography system using planar gradiometers: data collection and calibration Physiol. Meas. 27 S271-80

Safety I I C o E 2005 IEEE C95. 1-1992: IEEE standard for safety levels with respect to human exposure to radio frequency electromagnetic fields, $3 \mathrm{kHz}$ to $300 \mathrm{GHz}$ IEEE Stand.

Scharfetter H, Casañas R and Rosell J 2003 Biological tissue characterization by magnetic induction spectroscopy (MIS): requirements and limitations IEEE Trans. Biomed. Eng. 50 870-80

Scharfetter H, Merwa R and Pilz K 2005 A new type of gradiometer for the receiving circuit of magnetic induction tomography (MIT) Physiol. Meas. 26 S307-18

Tarjan P P and McFee R 1968 Electrodeless measurements of the effective resistivity of the human torso and head by magnetic induction IEEE Trans. Biomed. Eng. 15 266-78

Teichmann D, Foussier J and Leonhardt S 2010 Respiration monitoring based on magnetic induction using a single coil Biomedical Circuits and Systems Conf. vol 2 pp 37-40 (IEEE)

Teichmann D, Kuhn A, Leonhardt S and Walter M 2014 The MAIN shirt: a textile-integrated magnetic induction sensor array Sensors 14 1039-56

Walter M, Eilebrecht B, Wartzek T and Leonhardt S 2011 The smart car seat: personalized monitoring of vital signs in automotive applications Pers. Ubiquitous Comput. 15 707-15

Wartzek T, Weber H, Walter M, Eilebrecht B and Leonhardt S 2012 Automatic electrode selection in unobtrusive capacitive ECG measurements 25th Int. Symp. on Computer-Based Medical Systems pp 1-4

Watson S, Morris A, Williams R J, Griffiths H and Gough W 2004 A primary field compensation scheme for planar array magnetic induction tomography Physiol. Meas. $25271-9$

Wheeler H A 1928 Simple inductance formulas for radio coils Proc. Inst. Radio Eng. 16 1398-400

Wong M Y M, Pickwell-MacPherson E and Zhang Y T 2010 Contactless and continuous monitoring of heart rate based on photoplethysmography on a mattress Physiol. Meas. 31 1065-74 